\title{
The Use of Function/Means Trees for Modelling Technical, Semantic and Business Functions
}

\author{
Dr A.J. ROBOTHAM \\ Department of Control and Engineering Design \\ Technical University of Denmark, Building 358, DK - 2800 Lyngby, Denmark.
}

\section{SYNOPSIS}

This paper considers the feasibility of using the function/means tree to create a single tree for a complete motor vehicle. It is argued that function/means trees can be used for modelling technical and semantic functions, but it is an inappropriate method for business functions when one tree of the vehicle is required. Life cycle modelling provides an effective means for determining all the required purpose functions and is considered a more effective method than the function/means tree for this task when the structure and mode of operation of the vehicle is well defined and understood.

\section{INTRODUCTION}

In the continued pursuit of improved product quality, there is a necessity to design products that satisfy the quality requirements demanded by customers and product users. Furthermore, the product must also satisfy many other stakeholders, who in turn interact with the product at some time during its life cycle. Identifying the totality of stakeholder needs is not a trivial task. Whilst much attention has been given to understanding the so-called "voice of the customer", ensuring that the product development team is able to fulfil these needs by appropriate choices of functions, properties and product structuring is even more demanding. Design for quality demands that designers consciously create products with the appropriate functionality and properties to fulfil everybody's quality expectations.

\subsection{Background}

This paper has been inspired by a feasibility study that is being carried out with a well-known European motor vehicle manufacturer. The company wants a consistent approach for specifying new products and to ensure that all the design teams work towards the same specification during a new product development project. Furthermore, the company's products have well-known, characteristic qualities which define its so-called "Brand DNA"; these qualities must be carried over (and enhanced) to each new generation of products. 
The ambition of the company is to enhance the satisfaction of business, market and customer requirements by the product development teams and ensure a much greater focus on the product as a whole. The company wishes to counteract a phenomenon, which is reflected in Mørup's observation (1) of general design practice, that:

"Design engineers focus on the technology inside the product. He sees the product only as a technical challenge, a carrier of functions, and not as integral part of a system of: different users, working environments, life phases, and even society."

\subsection{Initial work by the company}

In its move towards product focused activities, the company has created a general list of functional requirements, which they believe captures all the requirements of any new vehicle. Furthermore, a more detailed function listing has been created from the general list by one of the design teams. The resulting document includes examples of alternative principal solutions for the functions the team has responsibility for. In this way, the document captures design ideas (whether they have been implemented in existing products or not) and links them to functional requirements.

However, the company seeks a systematic methodology for creating the functional requirement list and its link to principal solutions. The function/means tree allows relationships between functions and solutions, i.e. means, to be modelled. Consequently, it is perceived as being a candidate method for the ambition of having "one tree - one vehicle" to describe the functions and principal solutions of all vehicles.

The purpose of the study was to determine the feasibility of using the function/means tree for this task and to propose the methodology for its construction.

\subsection{Initial findings of the feasibility study}

Inspection of the company's general list of functional requirements showed that it had a hierarchical structure, which started with a statement of the overall purpose of the vehicle, e.g. a general function with conditions. The list was subsequently decomposed to at least four levels of sub-functions that included not only technical functions, as one would expect, but statements that could be classified as semantic and business functions. Furthermore, the functional requirement statements often included measures of performance, which described the goals to be achieved. Two consequences were immediately apparent:

- First, since the function/means tree has only been used for describing the technical functions of a technical system, the function/means tree method would have to be used outside the domain of any previous function/means tree modelling experience. Therefore, was it possible or appropriate to extend the use of function/means tree to describe semantic and business functional requirements? And if so, what implications does this have on the nature, structure and use of the function/means tree?

- Second, the function/means tree does not usually include measures of performance. Therefore, how can the function/means tree be modified to capture the completeness of the requirements in the general list? 


\section{MODELLING PRODUCTS}

During the product development process, a design team will utilise many different types of model to gain insight into the product that they are creating (2). The following is a concise review of three models, which provide the foundation for the discussion presented below.

\subsection{Life cycle modelling}

At the heart of Design for Quality is life cycle modelling (3), in which the "cradle-to-grave" life of a product is decomposed into discrete phases (e.g. production, assembly, distribution, sale, use, service, disposal) and discrete activities. Each life cycle phase may be modelled as a sequence of discrete transformation processes based upon the theory of technical systems (4). Scenarios are a useful means for modelling the activities. They enable the design team to empathise with the context of use and analyse the interactions between the product and customers, users and other stakeholders (5). From the analysis, the purpose of the product can be determined and the desired functionality and qualities defined.

\subsection{Domain Theory}

Andreasen's domain theory (6) provides insight into the relationship between the transformation processes that occur in the product life cycle and the elements of the product, which carry the required functionality and properties. There are three domains: transformation domain, organ domain, and parts domain (Figure 1).

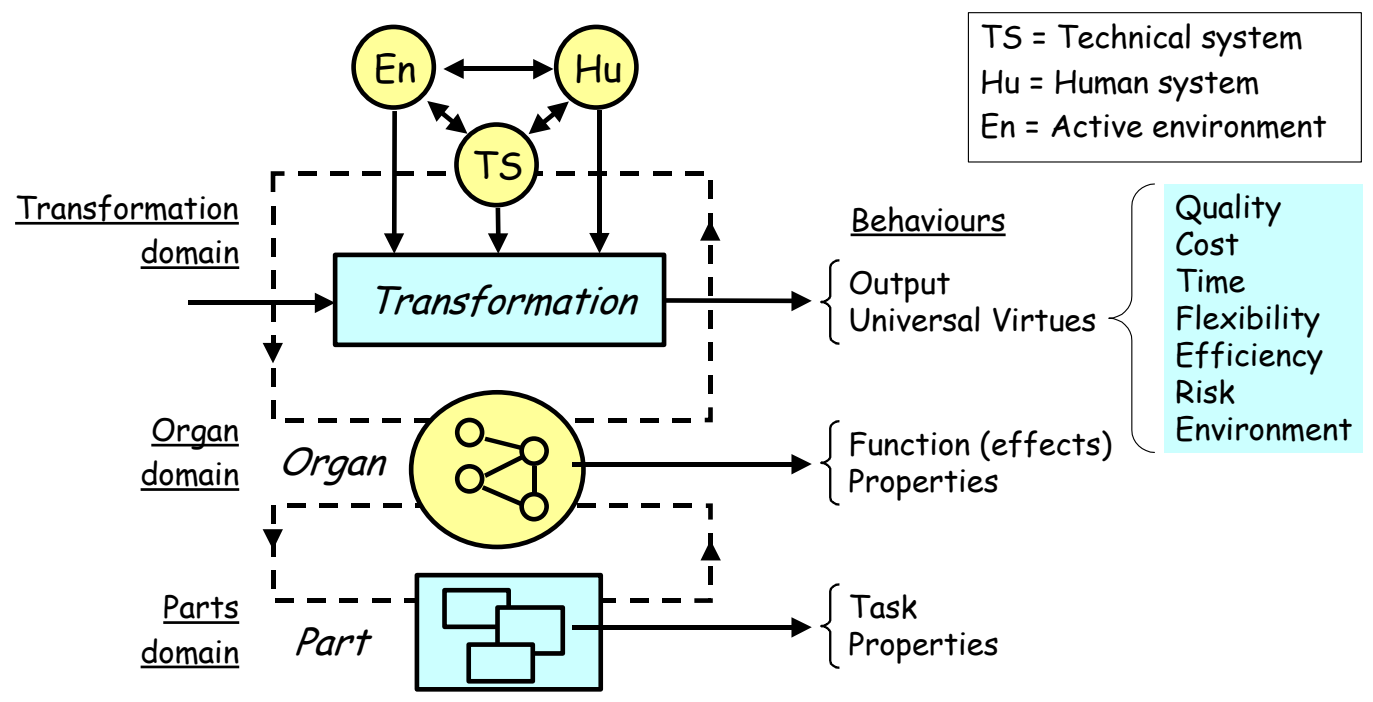

Figure 1: Domain theory (6)

In the transformation domain, there are interactions between the technical system (TS), the human operator $(\mathrm{Hu})$, the active environment $(\mathrm{En})$ and the operand, e.g. the thing that is being transformed. The operand is a passive member, whilst the $\mathrm{Hu}$, TS and En are active. The socalled 'seven universal virtues' (7) i.e. quality, time, cost, efficiency, flexibility, risk and environment, may be utilised to measure the effectiveness of each phase of the product life cycle, to compare alternative design solutions, or to clarify product development objectives.

An organ is an element of the functional structure of the technical system. The behaviour of an organ is characterised by the function it realises (e.g. the effect it creates) and its properties. A part is a unit of the anatomical structure of the TS. It is a carrier of elements of one or more organs. The observable properties of the TS depend upon the properties of parts. 


\subsection{Function Means Tree}

The function/means tree (8) is a method of modelling a product by the systematic decomposition of functions based upon the law of Hubka (4), which states there are causal relations between functions and means. Consequently, the function/means tree has a hierarchical structure of functions and means arranged on different levels and connected according to the causal relations (Figure 2). The top-level function is known as the purpose function. The function/means tree may also be used to show alternative solutions, from which an overall candidate solution maybe determined (Figure 2). Hansen provides a more complete description of the construction of function/means trees (9).

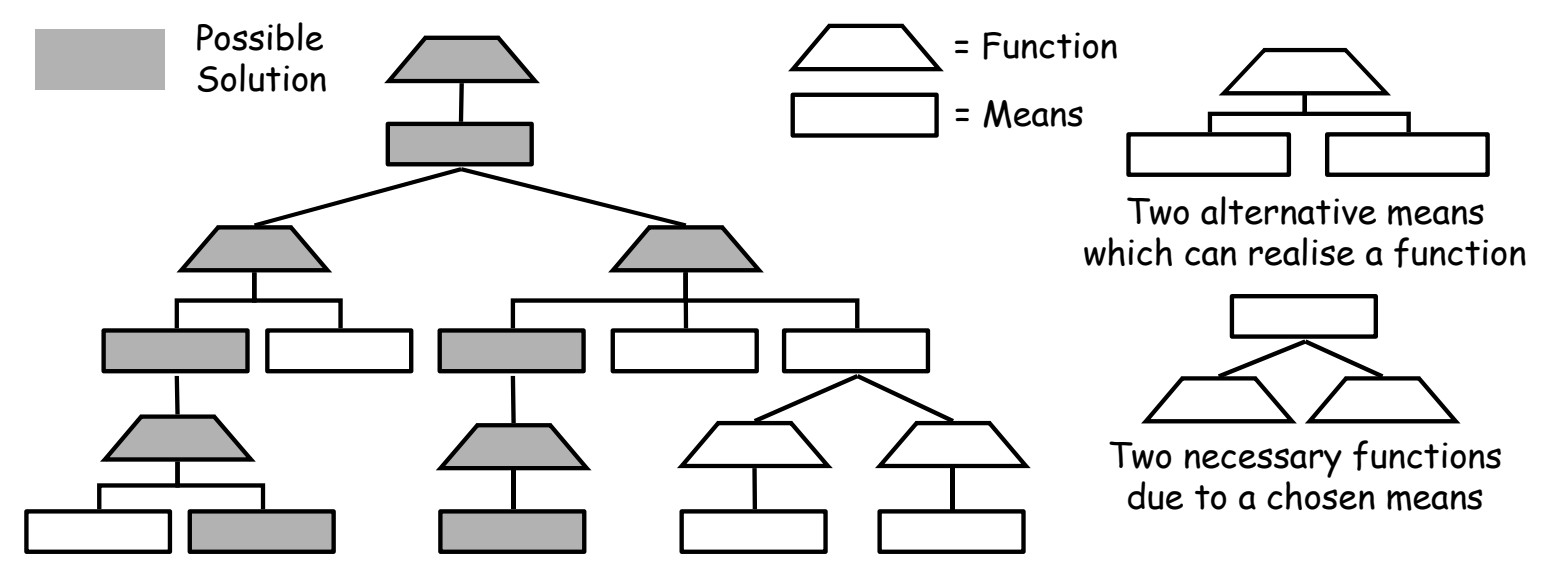

Figure 2: The principle structure of the function/means tree showing alternative solutions and a candidate solution [After Andreasen (8)]

\section{MODELLING PRODUCT FUNCTIONS}

In this section, the appropriateness of the function/means tree for modelling the technical, semantic and business functions will be discussed in turn and recommendations for any modifications to the modelling approach will be made.

\subsection{Technical Functions}

In the product modelling methods described in Section 2, technical functions are those realised by the technical system (TS) alone, e.g. the product. The transformation domain provides a great deal of flexibility for describing what goes on during the transformation process. We can divide the overall process into discrete sub-processes, in which a single function is performed. The overall effect may be realised by a series of sequential and simultaneous operations (Figure 3).

However, modelling the transformation by sub-processes assumes prior knowledge of the mode of action of the TS and its configuration (9). The company has a mature, well-defined product with very well understood modes of action. Consequently, they are able to create quite detailed models in the transformation domain without resorting to using function/means trees. The general functional requirement list is a result of a common knowledge in the company of all the functions that the vehicle must perform during its life based upon years of experience and a tacit knowledge of life cycle events.

The transformation domain shows both the TS and the human operator $(\mathrm{Hu})$ directly exerting effects on the operand of the transformation process. Consequently, it is necessary to 
determine which functions are performed by the TS and which by the Hu. The HumanMachine Interface (HMI) enables interactions to occur between the TS and the $\mathrm{Hu}$, e.g. for control and sequencing purposes.

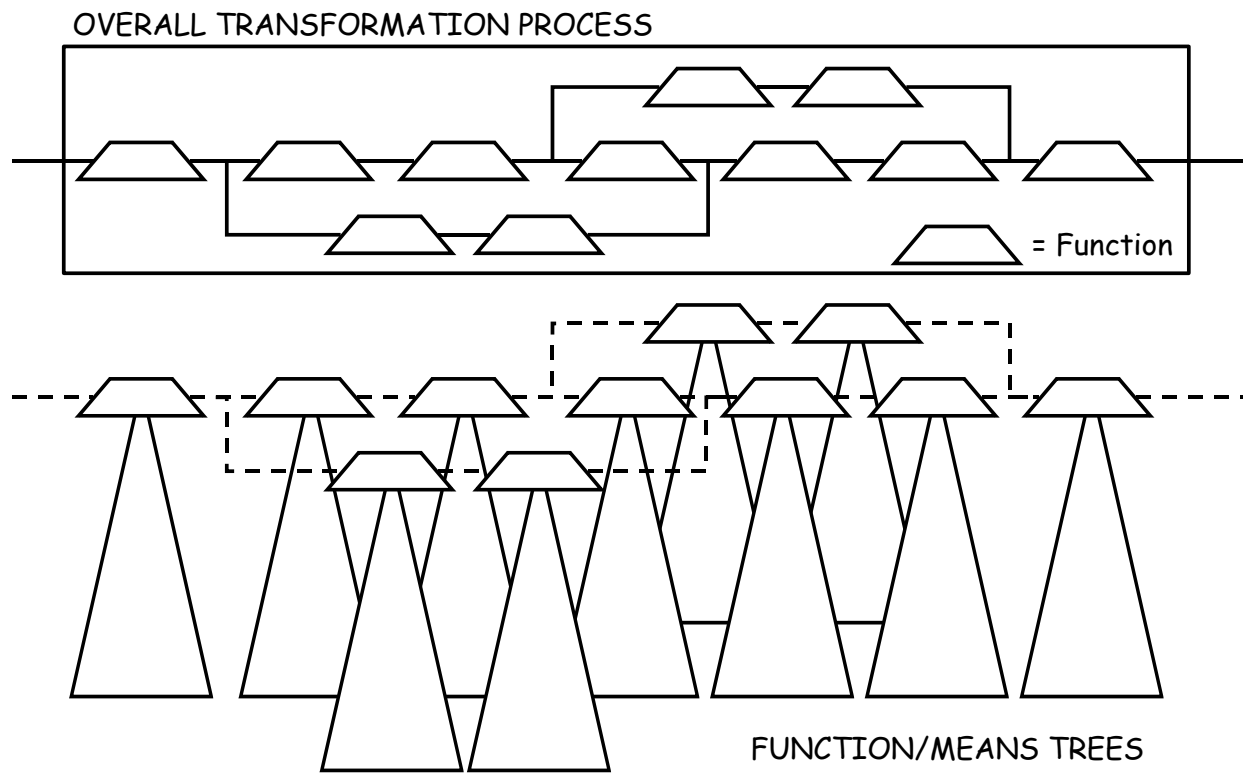

Figure 3: A transformation modelled as sub-functions with function/means trees.

With all the functions defined in the transformation domain and allocated to either the TS or the $\mathrm{Hu}$, function/means tree can be constructed for each function in turn. The result will be a series of function/means trees, which together show how the overall transformation process can be achieved (Figure 3). To date, the function/means tree has been exclusively used to describe how the technical system fulfils a function. Domain theory suggests we should limit the use of function/means tree to the organ domain, therefore the principal solutions shown in the tree will be organs or sub-organs.

The development of the function/means tree to the parts domain is not sensible. The parts domain is much more complex, since an individual component can be designed in many different ways and can carry elements from several different organs depending upon the configuration chosen by the designer, whereas an organ is uniquely related to a function. Thus limiting the function/means tree to the organ domain allows each purpose function to be uniquely represented by one tree. In principle, the same tree can be used whenever the purpose function is required in a transformation process or as a support function to a means.

\subsubsection{Proposed enhancements to the function/means tree}

If the function/means tree is to include alternative means, and provide a more complete model of the organ structure, then the tree needs to be supplemented by the configuration of the means (as proposed by Hansen (9)) and a listing of the functional properties of each means. The property list will show how well a specific means satisfies the function, e.g. to enable alternatives to be compared. These should be "tagged" directly to the means (Figure 4). Similarly, the configuration of a means will show what supporting functions are needed in the next level of the tree. Here, we may find that the TS requires a support function from either the $\mathrm{Hu}$ or the En. In this case, it would be useful if the function/means tree could show that the means comes from another system, which will require an interface of some kind. Once 
again, it would be helpful to include functional property list. This additional modification would assist the selection process, where the success of the function may depend significantly on the effect of elements outside the TS.

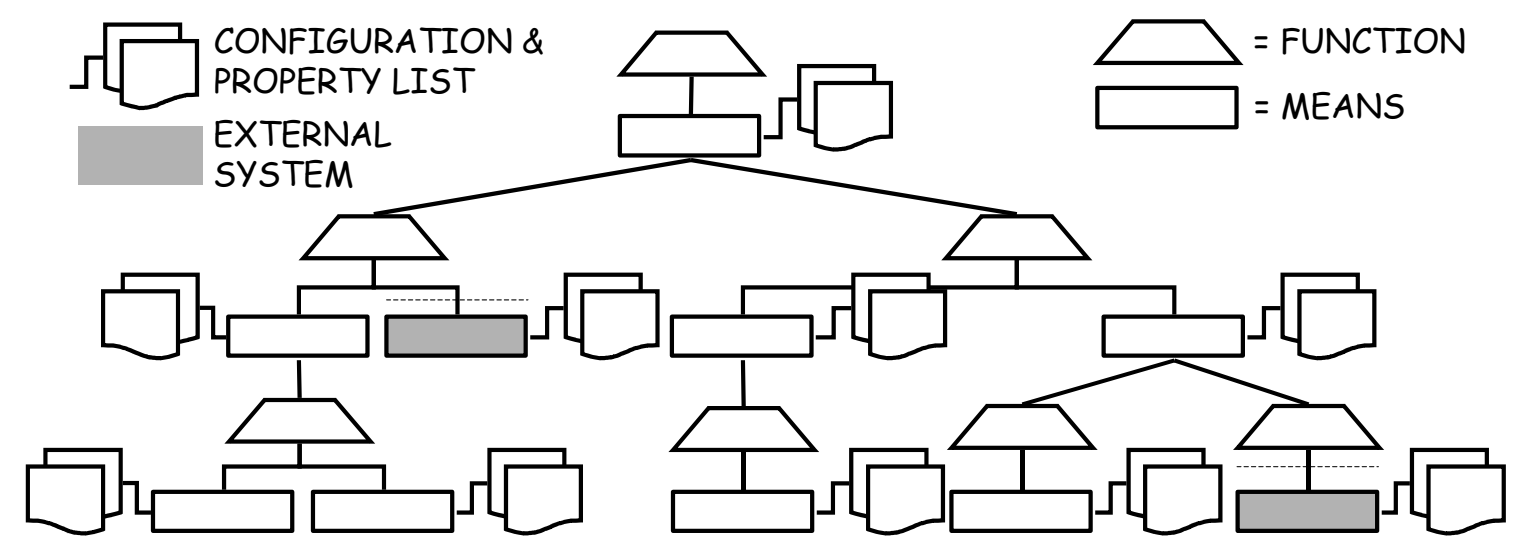

Figure 4: Functions/means tree with configurations, property lists and external means

\subsection{Semantic Functions}

The introduction of semantic functions into the general functional requirement list provides a challenge to the modelling methods described in Section 2, simply because their original focus was just on technical functions.

Since product quality is perceived when the customer interacts with the product and is strongly influenced by meta-products (10), products must clearly show their purpose, way of functioning, origin, kinship, properties, etc. The semantic function of a product is its ability to create signs, which will deliver specific messages about the product to the customer. Attention to semantic functions in design will yield products that are easier for people to understand and enhance the likelihood that its qualities are positively perceived by the customer. Monö proposes four semantic functions (10):

- To describe purpose, way of functioning, and using the product.

- To identify product, origin, and kinship.

- To exhort reactions.

- To express properties.

Semantic functions do not create active effects like technical functions, but influence perceptions in the mind of observers, e.g. customers. Consequently, semantic properties must be evident throughout the product, and especially so at human-machine interfaces. The TS is the means by which semantic functions are fulfilled. The recipients of the semantic messages are the humans in the transformation domain who interact with the product either in an active or passive way. For example, when a vehicle is being driven, the driver is the active $\mathrm{Hu}$ and a pedestrian standing at the kerb-side is a passive element of the En. In both cases, the product must provide appropriate messages to each person and create very clear ideas in their minds.

The concept of organs being function carriers is still valid when extended to semantic functions, e.g. "visual gestalts" (10) are examples of semantic organs. Therefore, it is feasible for semantic function/means trees to be constructed in the same manner as described in Section 3.1. The organs will be carriers of semantic related properties, which may often have 
to conform to statutory regulations or established norms. Norms will be heavily influenced by the meta-products the vehicle is related to. The integration of semantic and technical functions in a product model provides a more complete identification of product functions, remembering technical functions generate physical effects and semantic functions influence perceptions in the mind

\subsection{Business Functions}

The business functions identified in the general functional requirement list are not strictly physical functions that can be executed by the vehicle. The business functions, which were found to be closely to the vehicle, were specifications of product properties and performance goals, providing either specific targets or indicating the direction of change. Consequently, using the term "function" in this context is not consistent with its use in the language of product modelling. They are not functions carried by the technical system.

The theory of technical systems places "Management and Goal systems" in the active environment of the transformation domain. Their purpose is to give direction to the active elements and set goals for the overall transformation process. They influence the properties of the technical system to enable the transformation to be accomplished within the targets set by the management and goal system. Hansen (9) shows how goal specifications influence the decomposition procedure of a technical system in function/means tree construction.

Furthermore, among the business "functions" are those which refer to the non-use life phases of the vehicle, e.g. production, assembly, disposal, etc. In these life phases, the vehicle is the operand in the transformation process, has only a passive role and is acted upon by some other technical system, e.g. a manufacturing system. In these life phases it is only appropriate to assign properties to the vehicle - the functionality of the process is carried by the other technical systems. Therefore is not possible to create vehicle related function/means trees for these life phases, as the vehicle does not perform any functions.

The product properties, which are required as a result of business "functions", cannot easily be fitted into a function/means tree. Where the vehicle is active in the transformation process, it may be useful to modify the function/means tree to include the goal specification by "tagging" the function. However, the specification will be based upon the context of the transformation process. This would imply that the function/means tree would then become unique to the transformation in which the purpose function is identified. This conflicts with the opportunity to create generic trees that can be utilised repeatedly wherever the purpose function is demanded.

\section{CONCLUSIONS}

This feasibility study has considered the use of function/means tree for modelling a whole vehicle. The evaluation of its appropriateness has been based upon a consistent application of the theory of technical systems and domain theory. Life cycle modelling provides the starting point of the modelling task and the company has already used this approach to develop a general functional requirement list. The life cycle model is based upon knowledge of past and existing vehicles with well-known modes of operation, which may be unnecessary to represent in a function/means tree of the vehicle. The life cycle model will show when a purpose function is required and for what reason. 
The general functional requirement list includes a mixture of technical and semantic functions that can be assigned to the vehicle where it is an active element in a transformation process. In these situations, the function/means tree is a candidate method for modelling the realisation of both function types and for depicting principal solutions. However, to remain consistent with the domain theory, the means should be either organs or sub-organs and not parts. Modifying the function/means tree to include the configuration and property list of a means is advantageous, especially when developing generic trees for a purpose function in which alternative means are depicted. A further modification to the function/means tree would be to identify the means, which are external to the technical system.

The business "functions" were truly specifications of goals when considered from a product perspective. Consequently, they only influence the properties of the vehicle. Properties that are not directly related to a purpose function will not appear in a function/means tree, particularly if generic trees are required that are independent of context. Therefore business "functions" cannot be fully represented by a product related functions/means tree.

Finally, the original goal was "one tree - one vehicle" in which all functional requirements were assigned to the vehicle using a function/means tree. In reality the company requires "one tree - one business organisation" which will show how the company utilises all of its resources, i.e. means, to support the needs of all the stakeholders. This would require using the function/means tree in a new context and an alternative interpretation of its theory base.

\section{ACKNOWLEDGEMENTS}

The author is grateful for the inspiration of the company and my colleagues in IKS, but in particular the enthusiasm of Anders Norgaard and Jesper Tange in my discussions with them.

\section{REFERENCES}

1. Mørup, M., "Design for Quality", $\mathrm{PhD}$ thesis, Institute for Engineering Design, Technical University of Denmark, Lyngby, 1993.

2. Andreasen, M.M., "Modelling - The Language of the Designer", Journal of Engineering Design, Vol. 5, No. 2, 1994, pp 103-115.

3. Mørup, M., "Total life models - an important tool in Design for Quality". Proc. ICED93, The Hague, Netherlands, August 1993, pp 849-856.

4. Hubka, V. \& Eder, W:E., "Theory of Technical Systems: A Total Concept Theory for Engineering Design”, Springer-Verlag, Berlin Heidelberg, 1988.

5. Robotham, A.J. \& Hertzun, H., "Multi-Board Concept - A Scenario Based Approach for Supporting Product Quality and Life Cycle Oriented Design”, Proc. of Conf. Tools and Methods for Competitive Engineering 2000, The Hague, Netherlands, April 2000.

6. Andreasen, M.M., "Conceptual Design Capture", Engineering Design Conference 1998, Uxbridge, UK, 1998.

7. Olesen, J., "Concurrent Development in Manufacturing - Based on Dispositional Mechanisms", Integrated Production Systems, Technical University of Denmark, 1992.

8. Andreasen, M.M., "Machine Design Methods based on Systematic Approach Contribution to Design Theory", Doctoral Thesis, Lund University, Sweden, 1980.

9. Hansen, C.T., "An Approach to Simultaneous Synthesis and Optimization of Composite Mechanical Systems". Journal of Engineering design, Vol. 6, No. 3, 1995, pp 249-266.

10. Monö, R., "Design for Product Understanding: The Aesthetics of Design from a Semiotic Approach", Liber AB, Stockholm, 1997. ISBN 91-47-01105-X. 